\title{
Accuracy of routine laboratory diagnosis of malaria in the United Kingdom
}

\author{
L M Milne, M S Kyi, P L Chiodini, D C Warhurst
}

\begin{abstract}
Aims-To study the accuracy of routine laboratory diagnosis of malaria with the aim of improving accuracy in diagnosis in the future.

Methods-A comparative study was made of all blood films submitted to two laboratories in London providing a slidediagnostic service for malaria.

Results-There were 17 Plasmodium ovale infections, and of these only five (29.4\%) were correctly diagnosed by the submitting laboratory; whereas of 210 other single species infections, 162 $(77 \cdot 1 \%)$ were correctly diagnosed $\left(x^{2}=\right.$ $18 \cdot 4, \mathrm{p}<0 \cdot 0001)$. There were six patients with mixed infections; only one $(16.7 \%)$ was correctly diagnosed, whereas of 227 single species infections, 167 (73.6\%) were correctly diagnosed $(p=0.007$, using Fisher's exact test). There was no significant association between the presence of technical faults or numerous platelets and incorrect diagnosis.

Conclusions-Plasmodium ovale and mixed infections were diagnosed incorrectly significantly more often than other species. The presence of technical faults or numerous platelets had no significant effect on whether or not submitting laboratories correctly diagnosed malaria.
\end{abstract}

(F Clin Pathol 1994;47:740-742)

In 1991 there were 2332 cases of malaria reported in the United Kingdom with 12 deaths, 11 due to Plasmodium falciparum. ${ }^{1}$

The laboratory diagnosis of malaria is based on identifying parasites in peripheral blood by examination of thick and thin blood films. $^{2-5}$ Most slides made in the United Kingdom where malaria is considered a possible clinical diagnosis are submitted for verification to the Public Health Laboratory Service Malaria Reference Laboratory (MRL), or the Hospital for Tropical Diseases (HTD). In addition, HTD organises the United Kingdom National External Quality Assessment Scheme (UK NEQAS) for blood parasitology.

We have undertaken a comparative study of all blood films submitted to these two reference laboratories for confirmation or exclusion of malaria parasites. We attempted to find out the accuracy of routine laboratory diagnosis of malaria in the United Kingdom with the aim of improving accuracy in diagnosis in the future.

\section{Methods}

The study was conducted from 9 March to 31 May 1992 at the MRL and HTD. All blood films submitted during those dates for confirmation or exclusion of malaria were examined. Ninety one laboratories in the United Kingdom submitted blood films from a total of 267 patients. There were thick and thin blood films from 104 patients, thin films only from 161, and thick films only from two. A specimen was defined as the blood film/s received from one patient. The type of referring department was recorded. The malaria diagnosis made at the reference laboratories was defined as the true diagnosis and this was compared with the diagnosis made by the submitting laboratory as stated on the request form. Their diagnosis was regarded as correct if it stated the same malaria species as was reported by the reference laboratories, but incorrect if it did not, or if malaria parasites had been reported to be present by the submitting laboratory, but not seen by the reference laboratories.

Films which had been stained for the presence of malaria parasites before submission and those stained by the reference laboratories using standard methods ${ }^{3}$ were technically assessed. It was assumed that slides stained by the reference laboratories were stained correctly. The presence or absence of technical faults based on WHO criteria ${ }^{4}$ were recorded for $\mathrm{pH}$ of stain used (acidic, alkaline, appropriate), stain debris (present, absent), film spread on greasy slide (yes, no), fixation (poor, adequate), depth of thick film (too thin, too thick, appropriate), edge of spreader slide chipped (yes/no). Sequestrene effects (yes, no), which were defined as films containing crenated red blood cells or neutrophils showing vacuolation, swelling of the lobes of the nuclei, or disintegration ${ }^{5}$ were noted. The occurrence of individual instances or combinations of these faults was recorded. Thin blood films containing numerous platelets (more than five per field when examined microscopically at $\times 1000$ magnification) were also recorded.

Statistical analysis was done using Epi Info (Centers for Disease Control, Atlanta, Georgia). Tables were drawn up to test the null hypothesis that incorrect diagnoses from submitting laboratories were unrelated to the type of department submitting the specimen, the species of malaria diagnosed by the reference laboratories, or the presence of technical faults or films containing numerous platelets. 
treatment for $P$ falciparum differs from that of the other species infecting people. The case fatality rate due to $P$ falciparum is of the order of $1 \%$ in the United Kingdom ${ }^{10}$ and delay in diagnosis is a recognised factor contributing to a fatal outcome. In the case of $P$ vivax mortality in the United Kingdom due to this infection is negligible, ${ }^{10}$ but mistakes in laboratory diagnosis are still important, particularly if $P$ falciparum is diagnosed by the submitting laboratories, as primaquine, which is given to eliminate hypnozoites of $P$ vivax from the liver, is not required in $P$ falciparum infections. $P$ ovale was correctly diagnosed significantly less often than the other species $\left(\chi^{2}=18.4 ; \mathrm{p}<0.0001\right)$. For $P$ ovale, four infections were reported as $P$ vivax by the submitting laboratories. These two species are similar in appearance, ${ }^{9}$ and this may be the reason for the confusion. Fortunately, the treatment regimens would be identical. In 13 of 34 cases where malaria parasites were not seen by the reference laboratory malaria parasites were reported to be present or possibly present by the submitting laboratories. All species of malaria were reported, and a common error is the detection of malaria parasites where none is present. ${ }^{2}$

Table 2 shows that only one of six (16.7\%) mixed infections was correctly identified by the submitting laboratory. This was significantly less than for single strain infections ( $p=0.007$, using Fisher's exact test). However, $P$ falciparum was correctly identified in five of six mixed infections. There is a need for greater awareness by submitting laboratories of the possibility of infection with more than one species of malaria simultaneously, and the number of mixed species infections reported in the United Kingdom doubled between 1986 and 1989-90. ${ }^{11}$

Technical faults were sought, based on those identified by the WHO, ${ }^{4}$ and their effect on the diagnosis made by the submitting laboratory was examined. When examination for malaria parasites is undertaken, thin films are initially fixed in methanol before being stained. ${ }^{3}$ For 18 specimens this procedure had been performed poorly because their erythrocytes floated on the slide while we undertook microscopical examination. A common fault was films stained at an acidic $\mathrm{pH}$ - that is, $\mathrm{pH}$ 6.8-7 according to $\mathrm{WHO}^{4}$-which occurred in 50 specimens. The detection of malaria parasites in blood involves the use of differential stains which stain the nuclear material red and cytoplasm blue ${ }^{35}$ with clearly visible red blood cell stippling. ${ }^{35}$ These appearances will only occur if the $\mathrm{pH}$ used during staining is $\mathrm{pH} 7 \cdot 2,{ }^{3}$ as previous authors have emphasised. ${ }^{2}$ For example, Schuffner's dots due to $P$ vivax will not be seen if $\mathrm{pH} 6.8$ is used. Our results suggest that although routine haematology laboratories usually stain blood films at pH $6 \cdot 8,{ }^{2}$ some are inappropriately examining for the presence of malaria parasites at this pH. Stain debris was present on the blood films of 23 specimens. These artefacts may cause confusion in diagnosis ${ }^{4}$ and may be identified as malaria parasites. ${ }^{2}$ Sequestrene effects were seen in $85 \%$ of specimens exam- ined, which is to be expected as they occur within two to three hours of storage in EDTA. ${ }^{5}$ Despite the presence of various technical faults, they did not occur more commonly when submitting laboratories gave incorrect rather than correct diagnoses. Slides containing numerous platelets were not reported incorrectly more often than those without this feature. It is known that platelets, if superimposed on erythrocytes, may cause confusion in the diagnosis of malaria, ${ }^{2}$ and if several platelets are superimposed they may be mistaken for malaria parasites outside the red blood cell. ${ }^{9}$

Our study has shown that there is a need to improve the accuracy of routine laboratory diagnosis of malaria. This could be achieved if routine laboratories continue to gain experience by participating in external quality assurance schemes such as NEQAS. We have shown that their internal standard operating procedures may produce results which are technically faulty, as defined by WHO criteria. ${ }^{4}$ Although technical faults had no significant effect on the accuracy of diagnosis in our study, others ${ }^{24}$ have reported an effect. Laboratories may wish to discuss aspects of their standard operating procedures with designated experts, and must have good communications with their reference laboratories.

In conclusion, this study found that there were inaccuracies in the diagnosis of all species of malaria especially for $P$ ovale. The most serious inaccuracy was that five of 112 patients infected with $P$ falciparum were thought to be infected with another species. Only one of six mixed infections was accurately diagnosed and there was a lack of awareness of this diagnostic possibility by the submitting laboratories. The presence of various technical faults or numerous platelets did not differ significantly among submitting laboratories reporting correct or incorrect diagnoses.

We thank DJ Carucci and MA Quigley, both at the London School of Hygiene and Tropical Medicine, for providing statistical support during the analysis of this study. We also thank members of staff at the Public Health Laboratory Service Malaria Reference Laboratory and the Department of Clinica Parasitology, Hospital for Tropical Diseases for their cooperation.

1 Anonymous. The global problem of malaria. Communicable Diseases Report. 1992;2:227.

2 Chiodini PL, Moody AH. Techniques for the detection of malaria parasites. F Roy Soc Med 1989;82 (Supplement 17):41-3.

3 Fleck SL, Moody AH. Diagnostic techniques in medical parasitology. 1st edn. Cambridge: Wright, 1988:53-60.

4 World Health Organisation. Basic malaria microscopy. Part I. Learner's guide. Geneva: World Health Organisation, 1991.

5 Warhurst DC, Williams JE. Malaria and other bloodborne infections. In: Chanarin I, ed: Laboratory haematology. An account of laboratory techniques. $1 \mathrm{st}$ edn. Edinburgh: Churchill Livingstone, 1989:153-7.

6 Office of Population Censuses and Surveys Monitor. WR 92/10. London: OPCS, 1992:3.

7 Office of Population Censuses and Surveys Monitor. WR 92/22. London: OPCS, 1992:4.

8 Hawthorne M, Chiodini PL, Snell JJS, Moody AH, Ramsay A. Parasitology: United Kingdom National Quality Assessment Scheme. 7 Clin Pathol 1992;45: 968-74.

9 Bruce-Chwatt LJ. Essential malariology. 2nd edn. London: Heinemann Medical, 1985.

10 Bradley DJ. Current trends in malaria in Britain. $f$ Roy Soc Med 1989;82(Supplement 17):8-14.

11 Bradley DJ, Warhurst DC, Blaze M, Smith V. Malaria imported into the United Kingdom 1989 and 1990. Communicable Diseases Report 1991;1:45-8. 